\title{
Common seasonal respiratory virus infections in allogeneic stem cell transplant recipients during the SARS-COV-2 pandemic
}

\author{
Rosalía De la Puerta ${ }^{1}$ Juan Montoro ${ }^{1}{ }^{1}$ - Carla Aznar ${ }^{2}$ ' Ignacio Lorenzo ${ }^{1}$ - Eva María González-Barberá ${ }^{3}$. \\ Aitana Balaguer-Roselló ${ }^{1}$ - Manuel Guerreiro $\mathbb{1}^{1} \cdot$ Lara Domínguez $^{1} \cdot$ Miguel Salavert $^{4} \cdot$ Cristóbal Aguilar $^{1}$. \\ Javier de la Rubia ${ }^{1}$ - Jaime Sanz $\mathbb{1}^{1,2} \cdot$ María Dolores Gómez ${ }^{3} \cdot$ José Luis Piñana $\mathbb{C}^{1,5,6}$
}

Received: 1 February 2021 / Revised: 6 April 2021 / Accepted: 15 April 2021 / Published online: 4 May 2021

(c) The Author(s), under exclusive licence to Springer Nature Limited 2021

\begin{abstract}
The SARS-COV-2 pandemic has led to strict and generalized transmission prevention measures that may have changed the epidemiological landscape of common seasonal respiratory virus (CSRV). Through a prospective CSRV survey program conducted from 2016 onwards in allogeneic stem cell transplant (allo-HSCT) recipients with respiratory symptoms, we aimed to analyze and compare the epidemiology and characteristics of CSRV over three consecutive periods [from February 1 to September 30 of 2018 (P1), 2019 (P2), and 2020 (P3)]. CSRV screening was performed through multiplex PCR assays during the study period. We identified 188 consecutive allo-HSCT recipients with 406 episodes screened for CSRV during the study period, of which 147 developed 300 CSRV. In P1 and P2 we diagnosed 115 (38.3\%) and 145 (48.3\%) CSRV episodes, respectively, whereas in P3 only $40(13.3 \%)$ episodes were detected $(p<0.001)$. During P3, we observed a reduction of $80.2 \%$ in $\mathrm{Ev} / \mathrm{Rh}, 93.3 \%$ in RSV, $80 \%$ in hIV, 96.3\% HPIV, $68.4 \%$ in hMPV, $77.7 \%$ in ADV, $100 \%$ in $\mathrm{HBoV}$, and 53.6\% in $\mathrm{HCoV}$ as compared to $\mathrm{P} 1$ and $\mathrm{P} 2$. Consequently, we also observed a decline in absolute numbers of lower respiratory tract disease $(68.1 \%)$, co-infections $(91.7 \%)$, and hospitalizations $(72.6 \%)$ during P3. We diagnosed SARS-COV2 in nine allo-HSCT recipients, representing $23 \%$ of all CSRV detections in that period. In conclusion, we provide evidence of a significant drop in CSRV circulation during the SARS-COV-2 pandemic in our allo-HSCT recipients, indicating that prevention measures in the general population are highly effective in reducing CSRV prevalence and its complications in immunocompromised patients.
\end{abstract}

José Luis Piñana

jlpinana@gmail.com

1 Hematology Department, Hospital Universitari i Politècnic La Fe, Valencia, Spain

2 Outpatient Hematology/Oncology Nursing Unit, Hospital Universitari i Politècnic La Fe, Valencia, Spain

3 Microbiology Department, Hospital Universitari i Politècnic La Fe, Valencia, Spain

4 Department of Infectious Diseases, Hospital Universitari i Politècnic La Fe, Valencia, Spain

5 Hematology Department, Hospital Clinico Universitario de Valencia, Valencia, Spain

6 Fundación INCLIVA, Instituto de Investigación Sanitaria Hospital Clínico Universitario de Valencia, Valencia, Spain

\section{Highlights}

- Prevalence of common seasonal respiratory virus infections in allo-HSCT recipients has dropped during the SARS-COV-2 pandemic.

- Global preventive measures to limit SARS-COV-2 transmission may account for this marked reduction.

- The epidemiological landscape of common seasonal respiratory virus is changing.

\section{Introduction}

Health measures implemented worldwide to reduce transmission of severe acute respiratory syndrome coronavirus (SARS-COV-2) disease (COVID-19), including lockdowns, mask wearing, social distancing, school closures, travel restrictions, border closures, and comprehensive healthcare and patient protection measures such as COVID-19-free 
hospital areas, potentially also affect the transmission of other common seasonal respiratory viruses (CSRV). The initiation of COVID-19 restrictions in the Northern hemisphere coincided with the end of winter, with reports documenting a concurrent early end to local human influenza virus (hIV) and respiratory syncytial virus (RSV) seasons $[1,2]$. Likewise, restrictions implemented in Australia just prior to the Southern hemisphere winter, the usual period of peak local RSV and hIV infections, found $98 \%$ and 99.4\% reductions in RSV and hIV detections, respectively

[3] (https://www.who.int/emergencies/diseases/novel-corona virus-2019/interactive-timeline). Overall, it is fair to say that the preventive transmission measures implemented worldwide are a unique global phenomenon of this century, even despite the differing degrees of restrictions applied in each country. SARS-COV-2 did not show a seasonal predominance for some time, with the effect that preventive measures have expanded throughout the year in most countries, seemingly to last until herd immunity is achieved. Although CSRV has seasonal predominance during the cold months, many CSRVs still circulate in the community during warmer months and even year-round [4], posing serious health problems for high-risk patients, driven by direct or indirect CSRV effects. Applying strict and generalized preventive measures are likely to lead to lower incidence of other CSRVs, not only during the cold months but also beyond that season, in the general population worldwide and particularly in immunocompromised patients.

Current understanding supports that SARS-COV-2 and CSRV infections in recipients of allogeneic stem cell transplantation (allo-HSCT), mirror the incidence of infection in the community $[5,6]$. It is well-known that these infections are more severe than in the general population, showing a long viral shedding period, higher rates of progression from upper (URTD) to lower respiratory tract disease (LRTD) and higher mortality rates [6-8]. Alongside national and international transmission measures in the general population, early, more specific recommendations for transplant centers have been supplied by scientific societies to limit the transmission of SARS-COV-2 in allo-HSCT recipients [https://www.ebmt.org/sites/default/files/202012/EBMT\%20COVID-19\%20guidelines\%20and\%20summa ry\%20v.13.pdf]. In sum, it is likely that the COVID-19 pandemic could be associated with a significant reduction in other common CSRV infections frequently associated with allo-HSCT recipients. A useful resource to properly assess epidemiological changes of CSRVs in allo-HSCT recipients during the SARS-COV-2 pandemic are prospective CSRV survey programs, which enable comparison of CSRV prevalence across equivalent seasons over years.

The current study analyzes epidemiologic trends of CSRVs from February 1 to September 30, comparing three consecutive years $(2018,2019$, and 2020) in the setting of a prospective CSRV survey in allo-HCT recipients conducted at a single transplant center in Spain.

\section{Patients and methods}

\section{Study population and design}

This was a prospective cross-sectional study of respiratory virus infections in adult ( $>16$ years) allo-HCT recipients, limited to those with respiratory symptoms and being screened for CSRV infection throughout multiplex respiratory virus PCR assays through comparable periods (from February 1 to September 30) over three consecutive years $(2018,2019$, and 2020). The study sample was retrieved from our prospective CSRV survey registry conducted at the hematology division of the Hospital Universitari i Politècnic La Fe in Valencia, Spain. The local research ethics committee approved the study protocol in accordance with the Declaration of Helsinki and informed consent was obtained from patients.

\section{Cohort selection}

With the aim of comparing the CSRV prevalence over equivalent seasons across three consecutive years, we selected from our prospective CSRV database all consecutive allo-HSCT recipients with respiratory symptoms who were prospectively screened for CSRV respiratory infection during the study periods. We considered February 1 to September 30 as the recruitment season according to our local and national epidemiological data, since the first case of SARS-COV-2 in Spain was detected in La Gomera (Canary Islands) on January 31, 2020. The observation period ended on September 30, the date we closed the observations. On November 30 we updated the follow-up of all cases and started the analyses.

The first cohort included all consecutive allo-HCT recipients screened for CSRV between February 1, 2018 and September 30, 2018 [period 1 (P1)]. The second cohort comprised consecutive allo-HCT recipients screened for CSRV from February 1, 2019 to September 30, 2019 [period 2 (P2)], and finally a third cohort consisted of CSRV screening performed during the SARS-COV-2 pandemic between February 1, 2020 and September 30, 2020 [period 3 (P3)].

Only symptomatic respiratory virus infection episodes with laboratory-confirmed CSRVs and/or SARS-COV-2 were included in the epidemiological data of CSRV and SARS-COV-2 for each period. 


\section{CSRV survey characteristics and changes in clinical transplant practice during the COVID-19 pandemic}

From May 2016, we carried out a prospective CSRV survey in allo-HSCT recipients as previously described in detail elsewhere [9]. Allo-HCT recipients with respiratory symptoms underwent CSRV screening by multiplex PCR panels as a first-line test. Pre-COVID-19 pandemic, our protocol was to screen and attend recipients with respiratory symptoms in the outpatient transplant clinic during working hours. Transmission control measures were reinforced in the outpatient clinic following guidelines [10]. We boosted annual flu vaccination awareness information and prospectively collected clinical and laboratory variables at the time of CSRV screening [5].

On March 7, 2020, we diagnosed the first allo-HSCT recipient with COVID-19 at our transplant unit. On March 14, 2020 the Spanish authorities declared a nationwide state of emergency, which included severe restrictions limiting citizen mobility, such as school closures, borders closures, lockdowns, etc. Mask wearing has been mandatory in Spain since May 20, 2020. In early March, we implemented most of the COVID-19 recommendations from the EBMT [https://www.ebmt.org/sites/default/files/2020-12/ EBMT\%20COVID-19\%20guidelines\%20and\%20summary $\% 20$ v.13.pdf]. In brief, outpatient visits were substituted by telemedicine when appropriate and feasible [i.e., recipients at low-risk of infectious complications, such as those without signs/symptoms of active graft vs host disease (GvHD), not on immunosuppressive drugs, and with stable analytical parameters during the last outpatient visit]. Special emphasis was placed on respiratory symptoms questionnaires during phone calls with recipients. For necessary outpatient consultations, we implemented appropriate measures to reduce the risk of nosocomial transmission, such as visitor restrictions and proper protective equipment for physicians, nurses and recipients. Throughout the pandemic, recipients with respiratory symptoms attended physically or by phone call were prospectively screened for CSRVs (including SARS-COV-2) in a specific COVID-19 screening area or at the emergency unit of the hospital. In contrast, recipients without respiratory symptoms who were screened for SARS-COV-2 before any scheduled therapy (including the allo-HSCT procedure) or before hospital admission for any cause other than respiratory symptoms were not included in the current CSRV survey, since they were only screened for SARS-COV-2.

\section{CSRV respiratory infection definitions}

Upper respiratory tract disease (URTD) was defined as a combination of upper respiratory symptoms (rhinorrhea, sinusitis, otitis, or pharyngitis) as well as positive CSRV diagnosed by PCR test in the upper respiratory samples, and absence of lower respiratory tract disease (LRTD) symptoms and/or any indication of pulmonary infiltrates in chest X-ray or CT scan radiology results. We classified LRTD as possible, probable or confirmed, as previously described [11]. There were no probable episodes, as bronchoscopies were not performed in patients without radiological proof of pulmonary involvement.

\section{Technical and diagnostic considerations}

Patients with URTD and/or LRTD symptoms underwent nasopharyngeal aspiration, nasopharyngeal swabs or an induced sputum test, while BAL was performed in patients with radiological signs of LRTD whenever possible. Firstline CSRV screening was based on a PCR multiplex platform consisting of CLART $^{\circledR}$ PneumoVir DNA array assay (Genomica, Coslada, Spain). This RT-PCR can detect adenoviruses (ADVs); human bocavirus ( $\mathrm{HBoV}$ ); human coronavirus (HCoV) types 229E; hIV A A/H1N1, A/H3N2; hIV B and C; human metapneumovirus (hMPV); human parainfluenza virus (HPIV) 1, 2, 3, and 4; RSV A-B; and enterovirus/rhinovirus (Ev/Rh). From July 2018 onwards CSRV screening was performed by BioFire FilmArray ${ }^{\circledR}$ Respiratory Panel [BioFire Diagnostics (a bioMerieux company), Salt Lake City, UT]. This RT-PCR is able to detect 15 respiratory viruses: hIV types $\mathrm{A}, \mathrm{B}$, and $\mathrm{C}$ (with influenza A subtyping), ADV, HCoV HKU1, NL63, 229E and OC43, hMPV, EvRh, HPIV types 1-4 and RSV, and also detects four bacteria: Mycoplasma pneumoniae, Chlamydia pneumoniae and Bordetella pertussis and parapertusis. From February 1, 2020, we implemented CSRV screening by including SARS-COV-2 through several PCR assays, according to their availability, in respiratory samples from allo-HSCT recipients with respiratory symptoms.

\section{Endpoints and statistical analysis}

Our primary objective was to compare the overall epidemiology of CSRV infections in allo-HSCT recipients across the three periods. Secondary endpoints included descriptive analyses of specific CSRV characteristics between periods, as well as analysis of indicators of CSRV-related severity, such as LRTD prevalence, hospitalization rate, and allcause mortality.

Frequencies were compared using the $\chi^{2}$ test for categorical variables. Differences between medians were compared using the Kruskal-Wallis test. Two-sided exact $P$ values were reported and $P$-values $\leq 0.05$ were considered statistically significant. The data were analyzed with the SPSS (version 20.0) statistical package. 


\section{Results}

\section{Patient characteristics}

The study cohort included 188 allo-HSCT recipients with respiratory symptoms who were prospectively screened for CSRV through multiplex PCR platforms. Overall, $406 \mathrm{U} /$ LRTD episodes were tested for CSRV by PCR in the three periods. Of these 406 screened episodes, 300 (73.9\%) tested positive for one or more CSRV. Laboratory-confirmed CSRV involved 147 (78.2\%) out of 188 screened alloHSCT recipients.

Table 1 shows the main clinical and transplant characteristics of the whole cohort and those with laboratoryconfirmed CSRV. Median age was 50 years and $60.6 \%$ were male. The whole cohort included a high-risk population $(55.9 \%)$ since it included $27.7 \%$ unrelated donor recipients, $22.3 \%$ haploidentical family donor recipients, and $5.9 \%$ unrelated cord blood donor recipients.

\section{Clinical and epidemiological characteristics of CSRV episodes by screening period}

We performed 406 PCR tests overall: 145 (35.7\%) in P1, $187(46.1 \%)$ in $\mathrm{P} 2$, and $74(18.2 \%)$ in $\mathrm{P} 3(p<0.001)$. Of these, $115(79.3 \%), 145(77.5 \%)$, and $40(54 \%)$ tested positive for one or more CSRV, respectively $(p<0.001)$.

Table 2 summarizes CSRV infectious episodes by screening period. Laboratory-confirmed CSRV were significantly lower during P3. Although most characteristics showed no significant differences, allo-HSCT recipients developing a CSRV infection during P3 were more likely to be flu-vaccinated, and had lower rates of URTD symptoms, LRTD and CSRV co-infection $(p<0.05$ for all comparisons). In contrast, day-90 all-cause mortality rate was significantly higher during P3 $(p<0.001)$; in fact, three out of five deaths observed in that period were related to SARS-COV-2 (see Table 3).

In terms of CSRV episodes, we observed a $69.2 \%$ decrease during P3 compared to the average episodes from $\mathrm{P} 1$ and P2. Accordingly, we also observed reductions of $68.1 \%$ in LRTD occurrence, $91.7 \%$ in CSRV co-infections and $72.6 \%$ in hospital admissions in $\mathrm{P} 3$.

Comparing monthly CSRV episodes between periods, we observed a reduction of $48.2 \%$ and $38.1 \%$ in February and March, respectively, during P3. The drop was more pronounced from April to July 2020 (82.9\%, 100\%, 91.3\%, and $100 \%$, respectively). Finally, CSRV detection remained lower in August and September 2020 (77.7\% and 89.5\%, respectively) (Fig. 1).
Table 1 Patients and transplant's characteristics.

\begin{tabular}{|c|c|c|}
\hline Characteristics & $\begin{array}{l}(n=188) \\
\text { All patients }\end{array}$ & $\begin{array}{l}(n=147) \text { PCR- } \\
\text { positive patients }\end{array}$ \\
\hline Age (years), median (range) & $50(13.1-71)$ & $48.1(13.1-71)$ \\
\hline Male, $n(\%)$ & $114(60.6)$ & $84(57.1)$ \\
\hline \multicolumn{3}{|l|}{ Baseline disease, $n(\%)$} \\
\hline Myeloid diseases & $117(62.2)$ & $90(61.2)$ \\
\hline Lymphoid diseases & $62(33)$ & $50(34)$ \\
\hline Others & $9(4.8)$ & $7(4.8)$ \\
\hline Recipient CMV IgG positive, $n(\%)$ & $146(77.7)$ & $112(76.2)$ \\
\hline Donor CMV IgG positive, $n(\%)$ & $124(66)$ & 97 (67.4) \\
\hline \multicolumn{3}{|l|}{ Stem cell source, $n(\%)$} \\
\hline PB & $158(84)$ & $122(83)$ \\
\hline BM & $19(10.1)$ & $15(10.2)$ \\
\hline UC & $11(5.9)$ & $10(6.8)$ \\
\hline \multicolumn{3}{|l|}{ Type of donor, $n(\%)$} \\
\hline HLA-identical. related donor & $73(38.8)$ & $56(38.1)$ \\
\hline HLA-identical. unrelated donor & $52(27.7)$ & $40(27.2)$ \\
\hline Haploidentical family donor & $42(22.3)$ & $33(22.4)$ \\
\hline UCBT & $11(5.9)$ & $10(6.8)$ \\
\hline $\begin{array}{l}\text { HLA missmatch related or } \\
\text { unrelated donor }\end{array}$ & $10(5.3)$ & $8(5.4)$ \\
\hline \multicolumn{3}{|l|}{ Conditioning régimen, $n(\%)$} \\
\hline MAC & $90(47.9)$ & $75(51)$ \\
\hline \multicolumn{3}{|l|}{ GvHD prophylaxis, $n(\%)$} \\
\hline CsA-based regimen & $50(26.6)$ & $40(27.2)$ \\
\hline Post-Cy & $136(72.3)$ & $104(70.7)$ \\
\hline Others & $2(1.1)$ & $3(2.1)$ \\
\hline \multicolumn{3}{|l|}{ Prior GvHD, $n(\%)$} \\
\hline Acute & $92(48.9)$ & $71(48.2)$ \\
\hline Chronic & $127(67.6)$ & $109(74.1)$ \\
\hline \multicolumn{3}{|l|}{ Period of transplant, $n(\%)$} \\
\hline $2018-2020$ & $107(56.9)$ & $80(54.4)$ \\
\hline 2015-2017 & $58(30.8)$ & $46(31.3)$ \\
\hline 2012-2014 & $13(6.9)$ & $11(7.5)$ \\
\hline$\leq 2011$ & $10(5.4)$ & $10(6.8)$ \\
\hline \multicolumn{3}{|l|}{ Virus sampling periods, $n(\%)$} \\
\hline P1: Feb/2018-Sep/2018 & $145(35.7)$ & $115(38.3)$ \\
\hline P2: Feb/20219-Sep/2019 & $187(46.1)$ & $145(48.3)$ \\
\hline P3: Feb/2020-Sep/2020 & $74(18.2)$ & $40(13.3)$ \\
\hline Possible or proven CSRV LRTD, $n(\%)^{\mathrm{a}}$ & $52(27.7)$ & $43(29.3)$ \\
\hline IST during CSRV episode, $n(\%)$ & $124(66)$ & $91(61.9)$ \\
\hline Flu vaccination, $n(\%)$ & $64(34)$ & 49 (33.3) \\
\hline $\begin{array}{l}\text { Median days from allo-HSCT to CSRV } \\
\text { screening, (range) }\end{array}$ & $373(-4$ to 5422$)$ & 415 ( -4 to 5422$)$ \\
\hline \multicolumn{3}{|l|}{ Current status, $n(\%)$} \\
\hline Alive & 158 (83.6) & $124(84.4)$ \\
\hline Exitus & $31(16.5)$ & 23 (15.6) \\
\hline
\end{tabular}

$C M V$ cytomegalovirus, $U C B T$ unrelated cord blood transplant, $M A C$ mieloablative conditioning, $C s A$ cyclosporine A, Post-Cy posttransplant cyclophosphamide, allo-HSCT allogeneic hematopoietic stem cell transplantation, GvHD graft versus host disease, CSRV common seasonal respiratory virus, LRTD lower respiratory tract disease, IST immunosuppression systemic treatment.

a52 patients were suspected to have possible/probable LRTD and 43 patients had positive PCR test for one or more common seasonal respiratory virus/es in the upper and/or lower respiratory tract specimens. Among the 43 cases, 13 had possible and 30 proven LRTD, since the later had CSRV detected in BAL specimens. 
Table 2 Characteristics of CSRV by screening period in patients with positive PCR.

\begin{tabular}{|c|c|c|c|c|}
\hline & $\mathrm{P} 1$ & $\mathrm{P} 2$ & P3 & $P$-value \\
\hline PCR + episodes, $n(\%)$ & $115(38.3)$ & $145(48.3)$ & $40(13.3)$ & $P<0.001$ \\
\hline Median days from allo-HSCT to CSRV (range) & $260(-8$ to 4766$)$ & $275(-7$ to 3993$)$ & $473(-4$ to 5422$)$ & $P=0.015$ \\
\hline Flu vaccination, $n(\%)$ & $24(20.9)$ & $66(45.5)$ & $21(52.5)$ & $P<0.001$ \\
\hline IgG mg/dL, median (range) & $577(109-1350)$ & $662(0-14100)$ & $702(220-1716)$ & $n s$ \\
\hline Immunoglobulin support, $n(\%)$ & $15(13)$ & $19(13.1)$ & $7(17.5)$ & $n s$ \\
\hline HLA mismatched donor/recipient, $n(\%)$ & $45(39.1)$ & $51(35.2)$ & $12(30)$ & $n s$ \\
\hline Age $\geq 40, n(\%)$ & $71(61.7)$ & $115(79.3)$ & $31(77.5)$ & $P=0.005$ \\
\hline MAC regimen, $n(\%)$ & $72(62.6)$ & $70(48.3)$ & $23(57.5)$ & ns \\
\hline ALC $<0.5 \times 10 \mathrm{e} 9 / \mathrm{mL}, n(\%)$ & $27(23.5)$ & $28(19.3)$ & $6(15)$ & ns \\
\hline $\mathrm{ANC}<0.5 \times 10 \mathrm{e} 9 / \mathrm{mL}, n(\%)$ & $5(4.3)$ & $9(6.2)$ & $0(0)$ & ns \\
\hline Active GvHD, $n(\%)$ & $57(49.6)$ & $65(44.8)$ & $15(37.5)$ & ns \\
\hline Under IST, $n(\%)$ & $75(65.2)$ & $95(65.5)$ & $22(55)$ & ns \\
\hline Corticosteroids, $n(\%)$ & $30(26.1)$ & $32(22.1)$ & $6(15)$ & ns \\
\hline ISI (Low-moderate-high risk), n (\%) & $48-57-10(41.7-49.6-8.7)$ & $51-84-8(35.2-57.9-5.5)$ & $13-23-0(35-62.5-0)$ & ns \\
\hline Antibiotic therapy, $n(\%)$ & $82(71.4)$ & $105(72.4)$ & $30(75)$ & ns \\
\hline Fever $\left(>37.8^{\circ} \mathrm{C}\right), n(\%)$ & $47(40.9)$ & $62(42.8)$ & $20(50)$ & ns \\
\hline URTD, $n(\%)$ & $111(96.5)$ & $142(97.9)$ & $33(82.5)$ & $P<0.001$ \\
\hline LRTD, $n(\%)$ & $55(47.8)$ & $39(26.9)$ & $15(37.5)$ & $P=0.002$ \\
\hline CSRV co-infection, $n(\%)$ & $25(21.7)$ & $24(16.6)$ & $2(5)$ & $P=0.051$ \\
\hline Hospitalization, $n(\%)$ & $39(34)$ & $34(23.4)$ & $10(25)$ & \\
\hline Day-90 all-cause mortality, $n(\%)$ & $2(1.8)$ & $2(1.4)$ & $5(12.5)$ & $P=0.001$ \\
\hline Median follow-up after CSRV, days (range) & $720(14-950)$ & $426(7-580)$ & $138(5-224)$ & $P<0.001$ \\
\hline
\end{tabular}

$C S R V$ common seasonal respiratory virus, $+P C R$ positive polymerase chain reaction, allo- $H S C T$ allogenic hematopoietic stem cell transplant, $I g G$ immunoglobulin G, HLA major histocompatibility complex/human leukocyte antigen, MAC myeloablative, GvHD graft versus host disease, IST immunosuppressive therapy, ISI immunodeficiency scoring index, URTD upper respiratory tract disease, LRTD low respiratory tract disease, Max fever maximum temperature, $R C P$ reactive c protein, $A L C$ absolute lymphocytes count, $A N C$ absolute neutrophil count, $P 1$ period $1, P 2$ period 2 , $P 3$ period 3, ns not significant.

\section{Epidemiology and clinical characteristics of CSRV detections among periods}

Overall, we identified 355 CSRVs in 300 respiratory virus infection episodes. In 51 (17\%) of the episodes, we found more than one CSRV in the same respiratory sample. Epidemiological and clinical characteristics according to the three periods are shown in Table 3, in which the descriptive data of different CSRVs refer to individual CSRV detections irrespective of co-infection status.

Overall, the number of CSRV was significantly lower in P3 than P1 and P2 (Table 4) except for $\mathrm{HCoV}$ which rose in $\mathrm{P} 2$ and $\mathrm{P} 3$ compared to $\mathrm{P} 1$ (3.7\% and $7.9 \%$ vs $0.6 \%$; $p<$ $0.001)$. This latter finding was somewhat expected, as in July 2018 we introduced a novel PCR assay that included all four strains of $\mathrm{HCoV}$, in contrast to $\mathrm{P} 1$ where only HCoV 229E was included in the multiplex PCR assay from February 1 to June 30 2018. Finally, the reduction was $80.2 \%$ in $\mathrm{Ev} / \mathrm{Rh}, 93.3 \%$ in $\mathrm{RSV}, 80 \%$ in $\mathrm{hIV}, 96.3 \%$, in HPIV, $68.4 \%$ in hMPV, $77.7 \%$ in ADV, $100 \%$ in $\mathrm{HBoV}$, and $53.6 \%$ in $\mathrm{HCoV}$, the latter only compared with $\mathrm{P} 2$
(Fig. 2). During P3, except for one episode of $\mathrm{HCoV}$ in April and two Ev/Rh episodes in September, between April and September 2020 no other CSRVs were detected in our allo-HSCT recipients (Fig. 1). In contrast, SARS-COV-2 emerged in early March, and was still circulating in April, August, and September 2020. We diagnosed nine SARSCOV-2 cases during P3, which correspond to $23 \%$ of all CSRV episodes in that period.

\section{Discussion}

This study shows a remarkable reduction in absolute numbers (69.2\%) of CSRV respiratory infections during the SARS-COV-2 pandemic in a specific population, alloHSCT recipients with respiratory symptoms. The decrease was more pronounced from April 2020 onward and remained constant throughout the following months. Importantly, we also observed a dramatic fall in absolute numbers of LRTD (68.1\%), CSRV co-infections (91.7\%), and hospital admissions (72.6\%) during the COVID-19 
Table 3 Characteristics of respiratory infections according to common seasonal respiratory virus (CSRV) type and periods.

\begin{tabular}{|c|c|c|c|c|c|c|c|c|c|}
\hline \multirow{2}{*}{$\begin{array}{l}\mathrm{PCR}+=300 \text { peisodes } \\
\mathrm{N}^{\circ} \text { total } \mathrm{CSRV} \text { isolated }=355^{\mathrm{a}}\end{array}$} & \multicolumn{9}{|c|}{ Type of CSRV } \\
\hline & $\mathrm{Ev} / \mathrm{Rh}$ & hMPV & $\mathrm{ADV}$ & $\mathrm{HBoV}$ & RSV & hIV & HPIV & $\mathrm{HCoV}$ & $\begin{array}{l}\text { SARS- } \\
\text { CoV-2 }\end{array}$ \\
\hline \multicolumn{10}{|l|}{$\mathrm{P} 1\left(\mathrm{~N}^{\circ}\right.$ total $\mathrm{CSRV}$ isolated $\left.=140\right)$} \\
\hline Number of Episodes, $n(\%)$ & $50(14.1)$ & $16(4.5)$ & $2(0.6)$ & $6(1.7)$ & $19(5.4)$ & $23(6.5)$ & $22(6.2)$ & $2(0.6)$ & $0(0)$ \\
\hline URTD, $n(\%)$ & $48(96)$ & $15(93.8)$ & $2(100)$ & $6(100)$ & $18(94.7)$ & $22(95.7)$ & $22(100)$ & $2(100)$ & $0(0)$ \\
\hline LRTD, $n(\%)$ & $22(44)$ & $8(50)$ & $1(50)$ & $2(33.3)$ & $11(57.9)$ & $16(69.6)$ & $8(36.4)$ & $1(50)$ & $0(0)$ \\
\hline CARV Co-infection, $n(\%)$ & $8(16)$ & $4(25)$ & $1(50)$ & $5(83.3)$ & $12(63.2)$ & $12(52.2)$ & $6(27.3)$ & $0(0)$ & $0(0)$ \\
\hline Hospitalization, n (\%) & $17(34)$ & $2(12.5)$ & $1(50)$ & $2(33.3)$ & $10(52.6)$ & $11(47.8)$ & $6(27.3)$ & $1(50)$ & $0(0)$ \\
\hline Day-90 all-cause mortality & $0(0)$ & $0(0)$ & $0(0)$ & $0(0)$ & $1(5.3)$ & $0(0)$ & $1(4.5)$ & $0(0)$ & $0(0)$ \\
\hline \multicolumn{10}{|l|}{$\mathrm{P} 2\left(\mathrm{~N}^{\circ}\right.$ total CSRV isolated $\left.=171\right)$} \\
\hline Number of Episodes, $n(\%)$ & $61(17,2)$ & $3(0.8)$ & $7(2)$ & $2(0.6)$ & $11(3.1)$ & $27(7.6)$ & $32(9)$ & $28(7.9)$ & $0(0)$ \\
\hline URTD, $n(\%)$ & $60(98.4)$ & $3(100)$ & $7(100)$ & $2(100)$ & $11(100)$ & $26(96.3)$ & $32(100)$ & $28(100)$ & $0(0)$ \\
\hline LRTD, $n(\%)$ & $13(21.3)$ & $1(33.3)$ & $2(28.6)$ & $1(50)$ & $5(45.5)$ & $9(33.4)$ & $11(31.4)$ & $8(28.6)$ & $0(0)$ \\
\hline CARV Co-infection, $n(\%)$ & $15(24.6)$ & $0(0)$ & $3(42.9)$ & $1(50)$ & $4(36.4)$ & $5(18.6)$ & $11(31.4)$ & $10(35.7)$ & $0(0)$ \\
\hline Hospitalization, $n(\%)$ & $15(24.6)$ & $0(0)$ & $1(14.3)$ & $1(50)$ & $2(18.2)$ & $4(14.8)$ & $9(28.1)$ & $8(28.6)$ & $0(0)$ \\
\hline Day-90 all-cause mortality & $1(1.6)$ & $0(0)$ & $1(14.3)$ & $0(0)$ & $1(9.1)$ & $0(0)$ & $0(0)$ & $0(0)$ & $0(0)$ \\
\hline \multicolumn{10}{|l|}{ P3 $\left(\mathrm{N}^{\circ}\right.$ total CSRV isolated $\left.=44\right)$} \\
\hline Number of Episodes, $n(\%)$ & $11(3.1)$ & $3(0.8)$ & $1(0.3)$ & $0(0)$ & $1(0.3)$ & $5(1.4)$ & $1(0.3)$ & $13(3.7)$ & $9(2.6)$ \\
\hline URTD, $n(\%)$ & $8(72.7)$ & $3(100)$ & $1(100)$ & $0(0)$ & $1(100)$ & $5(100)$ & $1(100)$ & $13(100)$ & $5(55.6)$ \\
\hline LRTD, $n(\%)$ & $5(45.5)$ & $2(66.7)$ & $0(0)$ & $0(0)$ & $0(0)$ & $2(40)$ & $0(0)$ & $1(7.7)$ & $5(55.6)$ \\
\hline CSRV Co-infection, $n(\%)$ & $0(0)$ & $1(33.3)$ & $1(100)$ & $0(0)$ & $0(0)$ & $1(20)$ & $1(100)$ & $1(7.7)$ & $0(0)$ \\
\hline Hospitalization, $n(\%)$ & $3(27.3)$ & $2(66.7)$ & $0(0)$ & $0(0)$ & $0(0)$ & $1(20)$ & $0(0)$ & $0(0)$ & $4(44.4)$ \\
\hline Day-90 all-cause mortality & $1(9.1)$ & $0(0)$ & $0(0)$ & $0(0)$ & $0(0)$ & $0(0)$ & $0(0)$ & $1(7.1)$ & $3(33.3)$ \\
\hline
\end{tabular}

$C S R V$ common seasonal respiratory virus, $E v / R h$ Enterovirus/rhinovirus, $h M P V$ human metapneumovirus, $A D V$ adenovirus, $B C V$ bocavirus, $R S V$ respiratory syncytial virus, $h I V$ human influenza virus, $H P i V$ human parainfluenza virus, $H C o V$ human coronavirus, URTD upper respiratory tract disease, LRTD low respiratory tract disease, $P 1$ period $1, P 2$ period 2, $P 3$ period 3.

${ }^{a}$ The percentages of each CSRV episode were calculated using the total number of CSRV isolated as denominator.

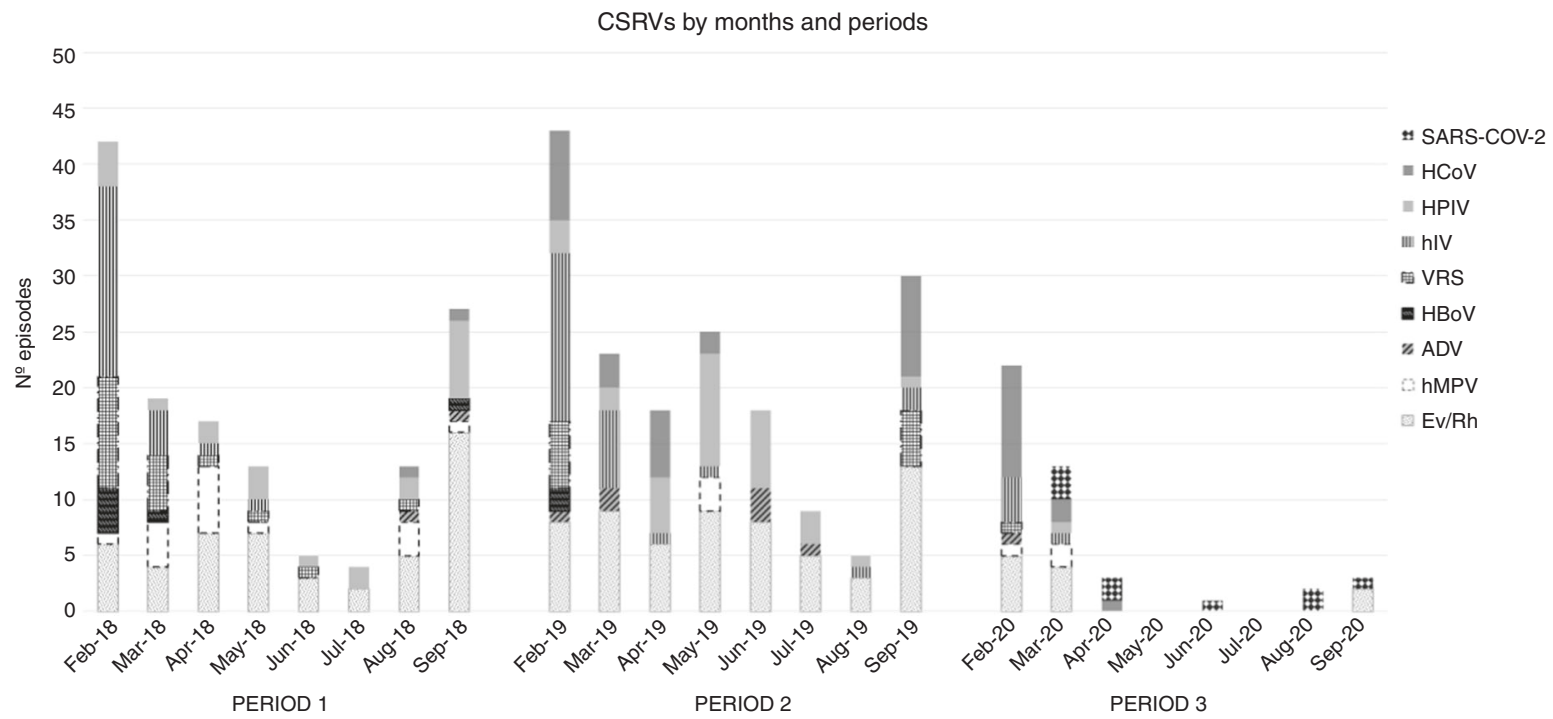

Fig. 1 Respiratory virus episodes. Prevalence of each common seasonal respiratory virus (CSRV) type by months and periods. 
Table 4 Summary of main types of CSRV analyzed by periods.

Fig. 2 Type of respiratory virus. Total common seasonal respiratory virus (CSRV) episodes by screening period.

\begin{tabular}{llllllllll}
\hline \multicolumn{1}{l}{ Type of CSRV } \\
\cline { 2 - 5 } & Ev/Rh & hMPV & ADV & HBoV & RSV & hIV & HPiV & HCoV & $\begin{array}{l}\text { SARS- } \\
\text { CoV-2 }\end{array}$ \\
\hline P1 & $50(14.1)$ & $16(4.5)$ & $2(0.6)$ & $6(1.7)$ & $19(5.4)$ & $23(6.5)$ & $22(6.2)$ & $2(0.6)$ & $0(0)$ \\
P2 & $61(17.2)$ & $3(0.8)$ & $7(2)$ & $2(0.6)$ & $11(3.1)$ & $27(7.6)$ & $32(9)$ & $28(7.9)$ & $0(0)$ \\
P3 & $11(3.1)$ & $3(0.8)$ & $1(0.3)$ & $0(0)$ & $1(0.3)$ & $5(1.4)$ & $1(0.3)$ & $13(3.7)$ & $9(2.6)$ \\
$p$-value & $P=0.01$ & $P=0.001$ & ns & ns & $P=0.014$ & ns & $P=0.017$ & $P<0.001$ & $P<0.001$ \\
\hline
\end{tabular}

$C S R V$ common seasonal respiratory virus, $E v / R h$ Enterovirus/rhinovirus, $h M P V$ human metapneumovirus, $A D V$ adenovirus, $B C V$ bocavirus, $R S V$ respiratory syncytial virus, $h I V$ human influenza virus, $H P i V$ human parainfluenza virus, $H C o V$ human coronavirus, $P 1$ period $1, P 2$ period $2, P 3$ period 3.

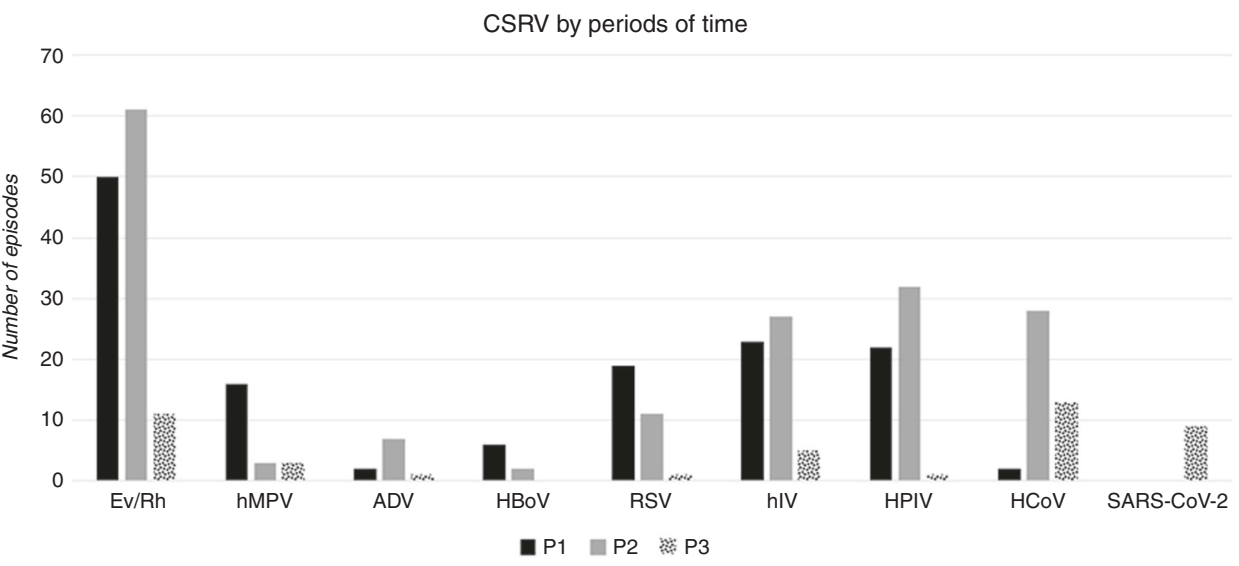

pandemic. In contrast, we noted a high number and rate of deaths during the pandemic, driven mainly by COVID-19.

Our prospective survey of CSRV in the allo-HSCT setting allowed us to compare epidemiological changes across time not only in hIV and RSV, but importantly also in most well-known CSRV causing moderate to severe respiratory infections in immunocompromised patients. We observed a marked reduction in CSRV starting in February 2020, before preventive measures were implemented in our country. In mid-March 2020, the Spanish authorities implemented strict measures to reduce community transmission of SARS-COV-2 and 2 weeks later (April 2020) there was a sharp decline $(82.9 \%)$ in CSRV infections in our allo-HSCT recipients. In May 20, 2020, wearing a mask was made compulsory in Spain. At this time, a $100 \%$ reduction in CSRV infections was achieved, highlighting the huge effect of such measures on CSRV circulation in the community. This effect has been already observed during the cold months in the northern and southern hemisphere, in particular for hIV and RSV [3, 12, 13]. Our study, however, was conducted from the end of winter and during warmer months, when hIV and RSV peaks have usually passed, supporting that transmission prevention measures also work outside the cold months for other CSRVs.

It could be argued that the fall in CSRV screening $(55.4 \%)$ during P3 was associated rather with the start of telemedicine, conducted more intensively at our center from
March 2020 to June 2020, which may have precluded attempts to match the pace of PCR testing seen in previous years. However, our allo-HSCT activity in the in patients and outpatients were not significantly different among periods. The total number of outpatient's medical visits was 1924 in P1 whereas it was 1821 in P2 and 2072 in P3. The number of allo-HSCT procedures among periods were also very similar, 50, 53, and 51, respectively. These facts support that the decrease or PCR tests in P3 was a direct consequence of a low burden of CSRV circulation. In addition, our epidemiological data are consistent with Spanish epidemiological data where hIV and RSV detections fall to zero at week 122020 [https://www.isciii.es/ QueHacemos/Servicios/VigilanciaSaludPublicaRENAVE/ EnfermedadesTransmisibles/Boletines/Documents/Boletin_ Epidemiologico_en_red/boletines\%20en\%20red\%202020/ IS_N\%C2\%BA\%2020-200512-WEB.pdf]. Other indicators also point to a decrease in CSRV circulation during P3, such as the lower probability of having a positive PCR test in this period (54\%) than in P2 and P1 $(79.3 \%$ and $77.5 \%$, respectively, $p<0.05)$. This is a well-characterized phenomenon, occurring when prevalence of CSRV in the community is low [14]. Furthermore, the reduction of CSRV detections translated into a lower number of other indirect markers such as LRTD, CSRV co-infections and CSRV-related hospitalizations, in line with other studies in the general population in the southern hemisphere $[15,16]$. 
Both facts point to lower CSRV circulation in the community during P3. Despite relaxation of transmission measures at the end of April 2020 (restaurants and businesses had largely reopened), CSRV circulation among allo-HSCT recipients was still absent until September 2020. In contrast, SARS-COV-2 reappeared in August 2020, indicating distinctive behavior and extraordinary efficacy in terms of community transmissibility compared to CSRV.

Regarding different types of CSRV, we observed a more pronounced reduction of Ev/Rh, hMPV, ADV, HBoV, RSV, hIV, and HPIV $(80.2 \%, 68.4 \%, 77.7 \%, 100 \%, 93.3 \%$, $80 \%$, and $96.3 \%$, respectively) than was seen with $\mathrm{HCoV}$ (53.2\% compared to P2). There is no obvious hypothesis as yet to explain these findings, although they are worth highlighting for future reference in the SARS-CoV-2 pandemic. Mortality was higher in P3 than P1 and P2, mainly due to SARS-COV-2 mortality in the most recent period.

Finally, our findings suggest that strict preventive measures in the whole population, not just the immunosuppressed population and care-givers, translate into reduced CSRV prevalence in immunocompromised patients. At the time of writing (January 31, 2021), neither hIV nor RSV infection has yet been detected in our allo-HSCT recipients. Therefore, this season's hIV vaccination resources could probably be allocated to the challenging task of administrating SARS-COV-2 vaccines to large proportion of the population in most countries.

The main limitation of this study is the use of two different PCR methods that differ minimally in analytical performance. The fact that asymptomatic recipients were not regularly screened, along with the use of telemedicine during P3, could also be viewed as drawbacks. Nonetheless, our prospective CARV survey mirrored national epidemiological data in influenza and RSV. In addition, the timespan of each period and inclusion of several CSRV should be considered strengths.

In conclusion, we provide evidence of a significant drop in CSRV activity during the SARS-COV-2 pandemic in our allo-HSCT recipients, supporting that prevention measures for general population are highly effective in reducing CSRV prevalence and its complications in immunocompromised patients. The findings of this study are likely generalizable to adults presenting with acute respiratory illness at a time of peak prevalence of SARS-COV-2 in developed healthcare systems.

\section{Compliance with ethical standards}

Conflict of interest The authors declare no competing interests.

Publisher's note Springer Nature remains neutral with regard to jurisdictional claims in published maps and institutional affiliations.

\section{References}

1. Cowling BJ, Ali ST, Ng TWY, Tsang TK, Li JCM, Fong MW, et al. Impact assessment of non-pharmaceutical interventions against coronavirus disease 2019 and influenza in Hong Kong: an observational study. Lancet Public Health. 2020;5:e279-e88.

2. Kuitunen I, Artama M, Mäkelä L, Backman K, Heiskanen-Kosma T, Renko M. Effect of social distancing due to the COVID-19 pandemic on the incidence of viral respiratory tract infections in children in Finland during early 2020. Pediatr Infect Dis J. 2020;39:e423-7.

3. Daniel K Yeoh, David A Foley, Cara A Minney-Smith, Andrew C Martin, Ariel O Mace, Chisha T Sikazwe, et al. The impact of COVID-19 public health measures on detections of influenza and respiratory syncytial virus in children during the 2020 Australian winter. Clin Infect Dis. 2020;28:ciaa1475.

4. Piñana JL, Madrid S, Pérez A, Hernández-Boluda JC, Giménez E, Terol MJ, et al. Epidemiologic and clinical characteristics of coronavirus and bocavirus respiratory infections after allogeneic stem cell transplantation: a prospective Single-Center Study. Biol Blood Marrow Transpl. 2018;24:563-70.

5. Piñana JL, Pérez A, Montoro J, Giménez E, Gómez MD, Lorenzo I, et al. Clinical effectiveness of influenza vaccination after allogeneic hematopoietic stem cell transplantation: a cross-sectional, prospective, observational study. Clin Infect Dis. 2019;68: 1894-903.

6. Piñana JL, Martino R, García-García I, Parody R, Morales MD, Benzo $G$, et al. Infectious complications subcommittee of the Spanish Hematopoietic Stem Cell Transplantation and Cell Therapy Group (GETH). Risk factors and outcome of COVID-19 in patients with hematological malignancies. Exp Hematol Oncol. 2020;9:21.

7. Chien JW, Martin PJ, Gooley TA, Flowers ME, Heckbert SR, Nichols WG, et al. Airflow obstruction after myeloablative allogeneic hematopoietic stem cell transplantation. Am J Respir Crit Care Med. 2003;168:208-14.

8. Erard V, Chien JW, Kim HW, Nichols WG, Flowers ME, Martin PJ, et al. Airflow decline after myeloablative allogeneic hematopoietic cell transplantation: the role of community respiratory viruses. J Infect Dis. 2006;193:1619-25.

9. Piñana J, Montoro J, Aznar C, Lorenzo I, Gómez MD, Guerreiro $\mathrm{M}$, et al. The clinical benefit of instituting a prospective clinical community-acquired respiratory virus surveillance program in allogeneic hematopoietic stem cell transplantation. J Infect. 2020;80:333-41.

10. Tomblyn M, Chiller T, Einsele H, Gress R, Sepkowitz K, Storek J. Guidelines for preventing infectious complications among hematopoietic cell transplant recipients: a global perspective. Biol Blood Marrow Transpl. 2009;15:1143-238.

11. Seo S, Xie H, Campbell AP, Kuypers JM, Leisenring WM, Englund JA. Parainfluenza virus lower respiratory tract disease after hematopoietic cell transplant: viral detection in the lung predicts outcome. Clin Infect Dis. 2014;58:1357-68.

12. Leuzinger K, Roloff T, Gosert R, Sogaard K, Naegele K, Rentsch $\mathrm{K}$, et al. Epidemiology of severe acute respiratory syndrome coronavirus 2 emergence amidst community-acquired respiratory viruses. J Infect Dis. 2020;222:1270-9.

13. Olsen SJ, Azziz-Baumgartner E, Budd AP, Brammer L, Sullivan $\mathrm{S}$, Pineda RF, et al. Decreased influenza activity during the COVID-19 pandemic-United States, Australia, Chile, and South Africa, 2020. Am J Transpl. 2020;20:3681-5.

14. Hanson KE, Azar MM, Banerjee R, Chou A, Colgrove RC, Ginocchio CC, et al. Molecular testing for acute respiratory tract infections: clinical and diagnostic recommendations from the 
IDSA's Diagnostics Committee. Clin Infect Dis. 2020;71: 2744-51.

15. Marriott D, Beresford R, Mirdad F, Stark D, Glanville A, Chapman S, et al. Concomitant marked decline in prevalence of SARS$\mathrm{CoV}-2$ and other respiratory viruses among symptomatic patients following public health interventions in Australia: data from St
Vincent's Hospital and associated screening clinics, Sydney, NSW. Clin Infect Dis. 2020;25:ciaa1256.

16. Kadambari S, Abo YN, Phuong LK, Osowicki J, Bryant PA. Decrease in infection-related hospital admissions during COVID19: why are parents avoiding the doctor? Pediatr Infect Dis J. 2020;39:e385-6. 